\title{
General sound classification and similarity in MPEG-7
}

\author{
MICHAEL CASEY \\ MERL Cambridge Research Laboratory, Cambridge, USA \\ E-mail: casey@merl.com
}

and MPEG layer III audio (MP3) standards have had in the past.

The MPEG-7 general sound recognition tools use decorrelated spectral features coupled with hidden Markov models (HMM) for computing similarity and generating source classifications. In addition to traditional timbre methods that apply only to isolated musical instrument notes, MPEG-7 also represents noise textures, environmental sounds, music recordings, melodic sequences, vocal utterances and sounds containing mixtures of sources. For some recent work in the area of sound indexing and retrieval, see Wold, Blum, Keislar and Wheaton (1996), Boreczky and Wilcox (1998), Martin and Kim (1998) and Zhang and Kuo (1998).

In this paper we shall discuss two new novel components of MPEG-7 audio. The first is the use of decorrelated spectral features for low-dimensional sound representation. The second component is source identification and general sound similarity using finite-state probabilistic inference models (see Casey and Westner 2000, Casey 2001a, b, c). These methods were chosen from a range of competing technologies and were found to exhibit good performance in a variety of applications. The tools will likely be useful to composers for automatically organising sonic materials using computational methods. The tools will also be of interest to software developers and researchers for building new advanced applications in music and audio processing.

\section{MPEG-7 CONTENT DESCRIPTION INTERFACE}

The MPEG-7 standard consists of descriptors and description schemes that are defined using a modified version of XML schema called the description definition language (DDL). A large number of descriptors have been defined covering images, audio, video and general multimedia usage. The DDL language ensures that media content description data may be shared between applications in much the same way that sound files are exchanged using standard file formats. For example, an audio spectrum is defined by a descriptor called AudiospectrumEnvelope. To use the descriptor, data is instantiated using the standardised DDL syntax. In this case, the spectrum data is stored 


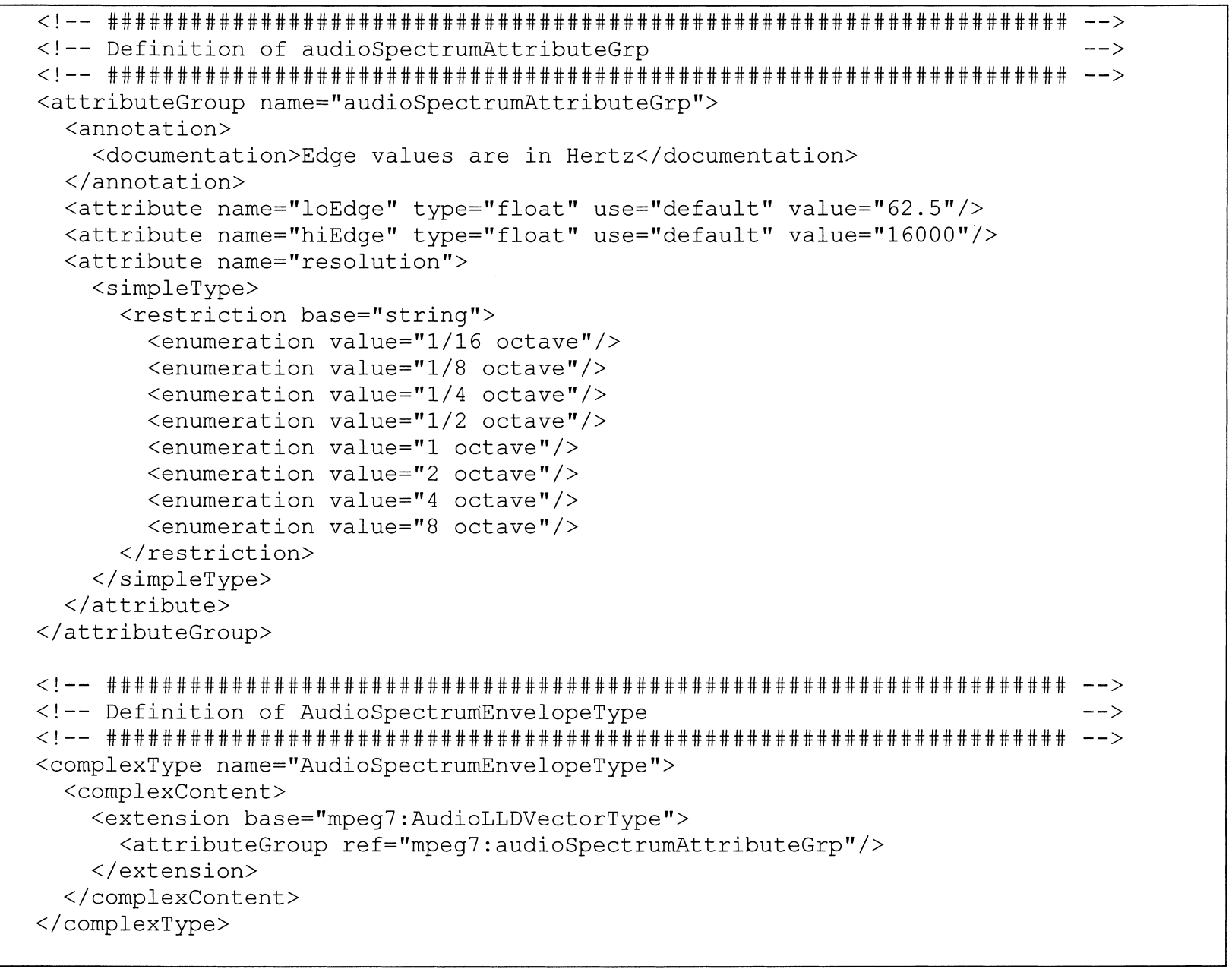

DDL Example 1. Standardised XML-Schema definition of an audio spectrum description.

as a series of vectors within the class (see DDL example 1). This descriptor enumerates allowable values for bands of logarithmically spaced power spectra. The spectrum values are contained in an encapsulated descriptor called AudioLLDVectorType (read audio low-level descriptor vector type) that is defined as a series of floating point numbers. The process for extracting values for the power spectrum is outside the scope of the standard, but guidelines are provided in order that implementers of the standard conform to certain constraints thus ensuring interoperability of descriptions generated by different implementations.

Figure 1 shows the distribution of power spectrum coefficients for a one-octave bandwidth power spectrum. In addition to the eight in-band coefficients, two additional coefficients enumerate the total power below and above the within-band limits, respectively.

\subsection{Decorrelated spectral features}

Spectrum-based features are often considered canonical for audio applications, but it is widely known that direct spectrum features are generally incompatible with classification applications due to their high dimensionality and their inconsistency. Each spectrum slice is an $n$-dimensional vector, with $n$ being the number of spectral channels; therefore, typical values of a linearly-spaced spectrum are between 64 and 1,024 dimensions. Probability classifiers require relatively low-dimensional data representations, preferably fewer than 10 dimensions. A logarithmically spaced frequency spectrum, such as the one-octave bandwidth power spectrum shown in figure 1 , reduces the dimensionality of the representation significantly, but necessarily disregards much information due to the low-frequency resolution. What is required is a representation that makes a compromise between dimensionality reduction and information loss.

A well-known technique for reducing the dimensionality of data whilst retaining maximum information is to use data-derived basis functions, such as computed by principal component analysis (PCA), singular value decomposition (SVD) or independent component analysis (ICA). A spectrogram may be reconstructed using a set of decorrelated frequency basis functions derived using one of these methods. Fewer functions are 


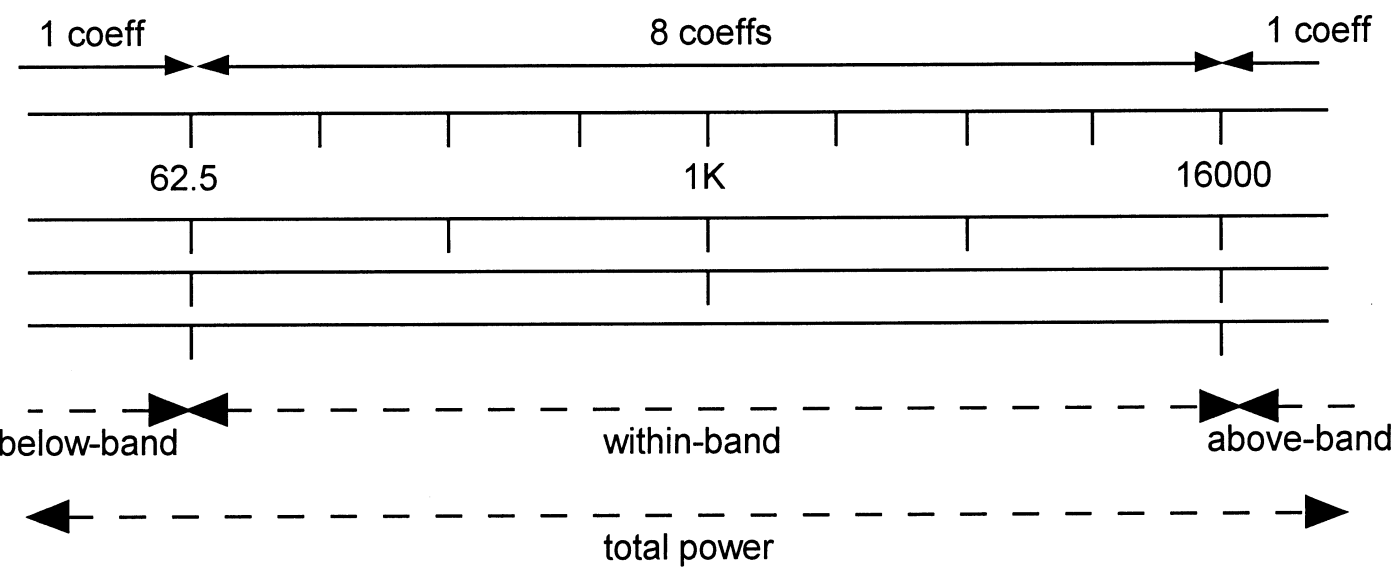

Figure 1. Illustration of audio spectrum envelope bands for one-octave bandwidth resolution.

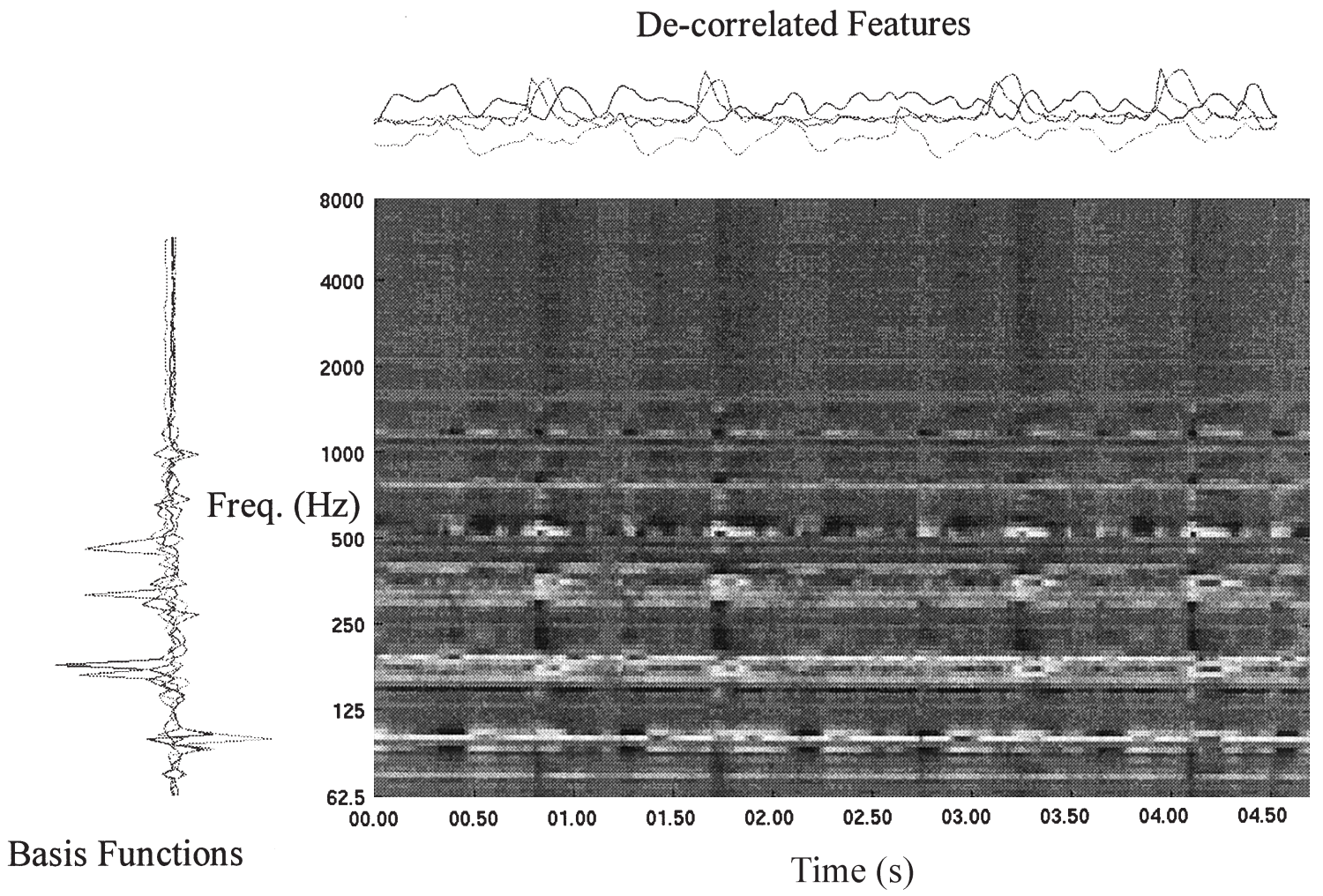

Figure 2. Fitting a spectrogram with data-derived basis functions; in this case the functions were derived from the spectrum using a singular value decomposition (SVD).

required to reconstruct a given spectrogram than the total number of frequency channels, hence the possibility for dimensionality reduction. For example, figure 2 shows a spectrum of five seconds of pop music reconstructed using only four basis functions. The functions on the right of the figure are the frequency basis functions, those above the figure are the reduced dimension features used for classification. In this case, $70 \%$ of the original 32-dimensional data is represented by only the 4-dimensional functions.

The AudiospectrumBasis descriptor contains basis functions that are used to project high-dimensional spectrum descriptions into a low-dimensional representation contained by the AudiospectrumProjection descriptor (see DDL example 2). The reduced bases consist of decorrelated features of the spectrum with the important information described much more efficiently than the direct spectrum representation. This reduced representation is well suited for use with probability model classifiers that typically perform best when the input features consist of fewer than 10 dimensions. These features were found to exhibit superior performance in sound recognition tasks and, thus, we shall describe their extraction and use below. 


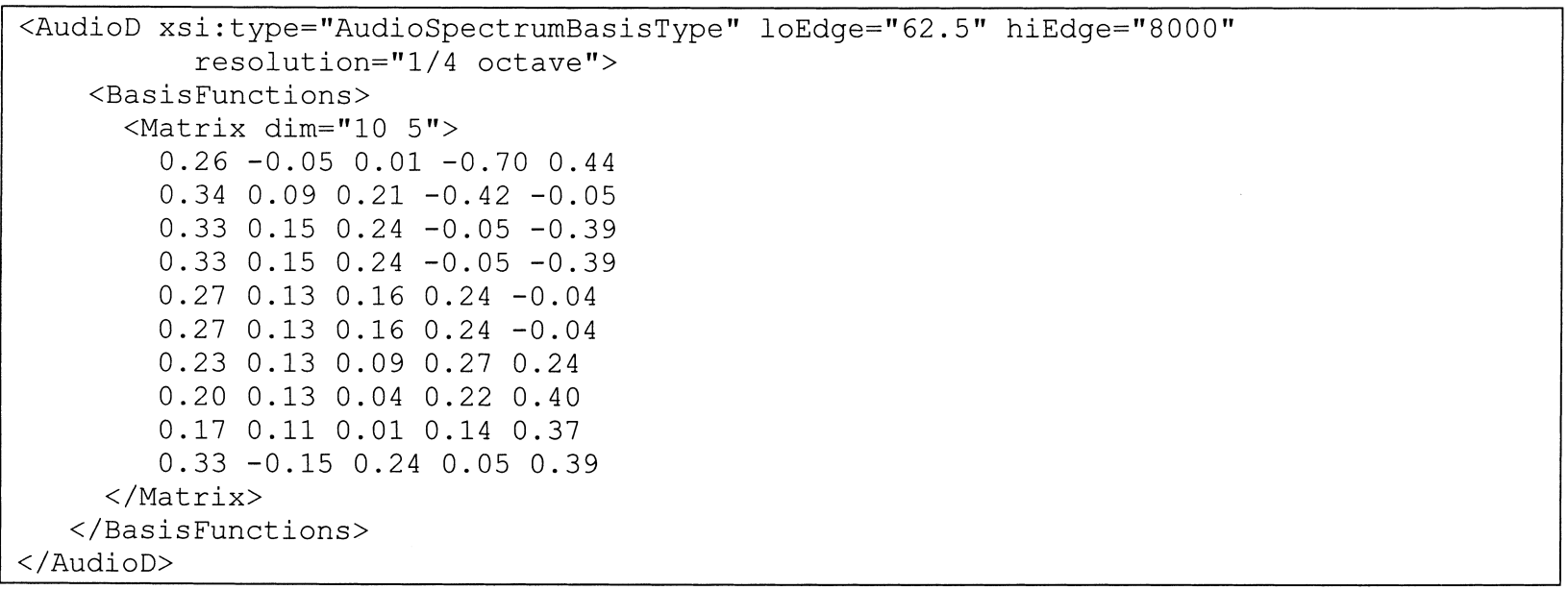

DDL Example 2. Description of five basis functions using AudiospectrumBasisType. The description definition language is based on XML schema with some extensions specific to MPEG-7. (The floating-point resolution has been truncated for clarity.)

\subsection{Spectrum basis function extraction method}

The extraction method for AudiospectrumBasis and AudiospectrumProjection is detailed within the MPEG-7 standard. It is considered that these steps must be used in extracting a reduced-dimension description in order to conform to the standard. Within each step there is opportunity for alternate implementations. As such, the following procedure outlines the standardised extraction method for ISA basis functions:

(1) Power spectrum. Instantiate an Audiospectrum Envelope descriptor using the extraction method defined in AudioSpectrumEnvelopeType. The resulting data will be a SeriesOfVectors with $M$ frames and $N$ frequency bins.

(2) Log-scale norming. For each spectral vector, $\mathbf{x}$, in AudioSpectrumEnvelope, convert the power spectrum to a decibel scale,

$$
\mathbf{z}=10 \log _{10}(\mathbf{x})
$$

and compute the L2-norm of the vector elements:

$$
r=\sqrt{\sum_{k=1}^{N} z_{k}^{2}} .
$$

The new unit-norm spectral vector is calculated by:

$$
\tilde{\mathbf{x}}=\frac{\mathbf{z}}{r} \text {. }
$$

(3) Observation matrix. Place each vector row-wise into a matrix. The size of the resulting matrix is $M \times N$ where $M$ is the number of time frames and $N$ is the number of frequency bins. The matrix will have the following structure:

$$
\tilde{\mathbf{X}}=\left[\begin{array}{c}
\tilde{\mathbf{x}}_{1}^{T} \\
\tilde{\mathbf{x}}_{2}^{T} \\
\vdots \\
\tilde{\mathbf{x}}_{M}^{T}
\end{array}\right]
$$

(4) Basis extraction. Extract a basis using a singular value decomposition (SVD); commonly implemented as a built-in function in many software packages using the command $[\mathrm{U}, \mathrm{S}, \mathrm{V}]=$ $\operatorname{SVD}(\mathrm{X}, 0)$. Use the economy SVD when available since the row-basis functions are not required and this will increase extraction efficiency. The SVD factors the matrix from step (3) in the following way:

$$
\tilde{\mathbf{X}}=\mathbf{U S V}^{T},
$$

where $\mathbf{X}$ is factored into the matrix product of three matrices; the row basis $\mathbf{U}$, the diagonal singular value matrix $\mathbf{S}$, and the transposed column basis functions $\mathbf{V}$. Reduce the basis by retaining only the first $K$ basis functions, i.e. the first $K$ columns of $\mathbf{V}$ :

$$
\mathbf{v}_{K}=\left[\begin{array}{lll}
\mathbf{v}_{1} \mathbf{v}_{2} \ldots & \mathbf{v}_{k}
\end{array}\right] .
$$

$K$ is typically in the range of 3-10 basis functions for feature-based applications. To calculate the proportion of information retained for $K$ basis functions, use the singular values contained in matrix $\mathbf{S}$ :

$$
I(K)=\frac{\sum_{i=1}^{K} S(i, i)}{\sum_{j=1}^{K} S(j, j)},
$$

where $I(K)$ is the proportion of information retained for $K$ basis functions, and $N$ is the total number of basis functions which is also equal to the number of spectral bins. The SVD basis functions are stored in 
the columns of a matrix within the AudiospectrumBasistype descriptor.

(5) Statistically independent basis (optional). After extracting the reduced SVD basis, $\mathbf{V}$, a further step consisting of basis rotation to directions of maximal statistical independence is often desirable. This is necessary for displaying independent components of a spectrogram and for any application requiring maximum separation of features.

To find a statistically independent basis using the basis functions obtained in step (4), use one of the wellknown, widely published independent component (ICA) algorithms such as INFOMAX, JADE or FastICA (Bell and Sejnowski 1995, Cardoso and Laheld 1996, Hyvarinen 1999).

The ICA basis is the same size as the SVD basis and is stored in the columns of the matrix contained in the AudiospectrumBasistype descriptor. The retained information ratio, $I(K)$, is equivalent to the SVD when using the given extraction method.

\subsection{Basis projection features}

Figure 3 shows the extraction system diagram for both AudiospectrumBasis and AudiospectrumProjection. The basis projection gives time masking functions that are combined with the spectrum basis functions to reconstruct independent spectrogram components. To perform extraction for SpectrumBasisProjection, follow steps (1)-(3) described above for AudiospectrumBasis extraction; this produces a spectrum matrix. The only further requirement is to multiply the spectrum matrix with the basis vectors obtained in step (4) or, optionally, step (5). The method is the same for both SVD and ICA basis functions:

$$
\tilde{\mathbf{Y}}_{k}=\tilde{\mathbf{X}} \overline{\mathbf{V}}_{k}
$$

where $\mathbf{Y}$ is a matrix consisting of the reduced dimension features after projection of the spectrum against the basis V. For independent spectrogram reconstruction, extract the non-normalised spectrum projection by skipping the normalisation step (2) in AudiospectrumBasis extraction. Thus:

$$
\mathbf{Y}_{k}=\mathbf{X} \overline{\mathbf{V}}_{k} \text {. }
$$

Now, to reconstruct an indpendent spectrogram component, use the individual vector pairs corresponding to the $K$ th vector in AudiospectrumBasis and AudiospectrumProjection, and apply the reconstruction equation:

$$
\mathbf{X}_{k}=\mathbf{y}_{k} \overline{\mathbf{v}}_{k}^{+},
$$

where the + operator indicates the transpose for SVD basis functions (which are orthonormal) or the pseudoinverse for ICA basis functions (non-orthogonal).

The method outlined above represents a powerful tool that can be used for many purposes. The extracted sources may be subjected to further analysis such as tempo estimation, rhythm analysis or fundamental frequency extraction. For example, we now consider how ISA features may be used for sound recognition and similarity judgements for general audio.

\section{GENERALISED SOUND RECOGNITION}

A number of tools exist within the MPEG-7 framework for computing similarity between segments of audio. In this section we describe tools for representing category concepts as well as tools for computing similarity in a general manner. The method involves training statistical models to learn to recognise the classes of sound defined in a taxonomy.

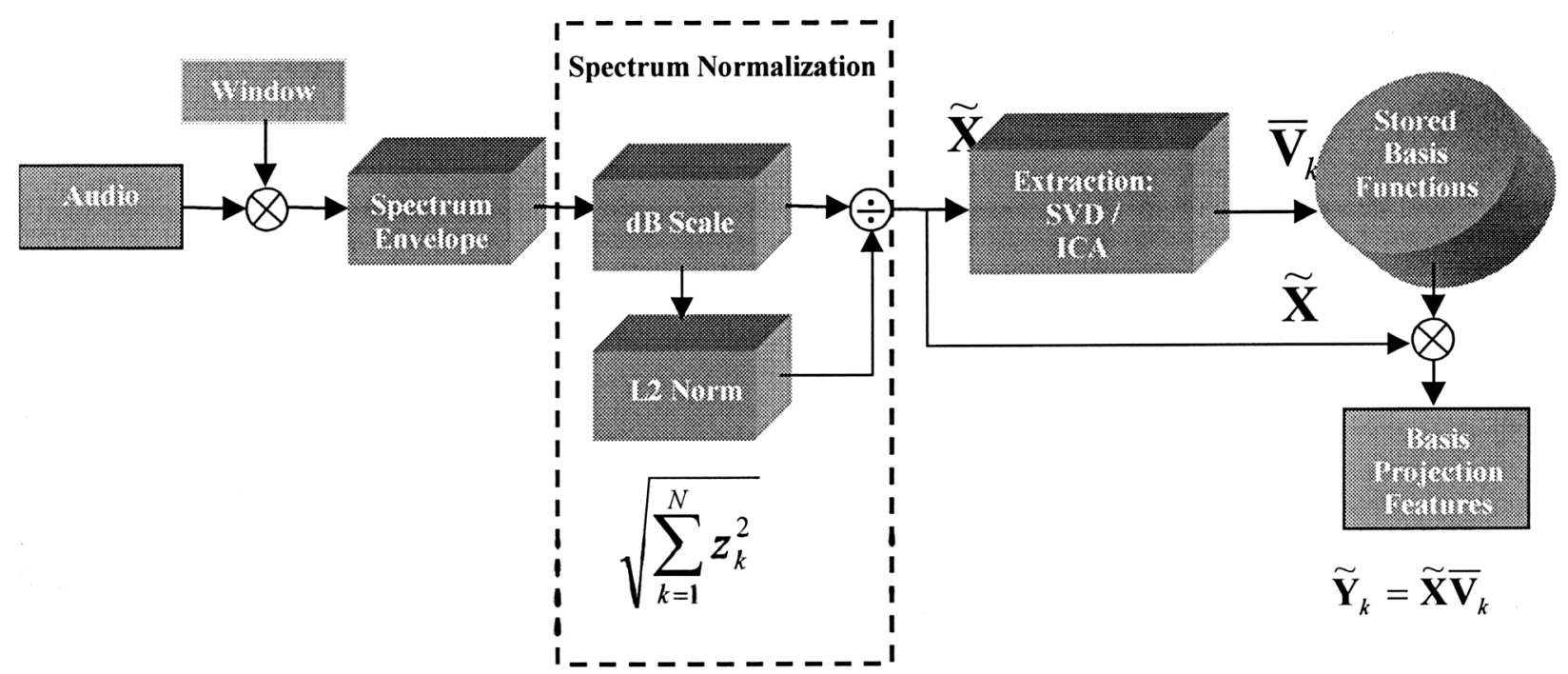

Figure 3. Extraction method for AudiospectrumBasis and AudiospectrumProjection. 


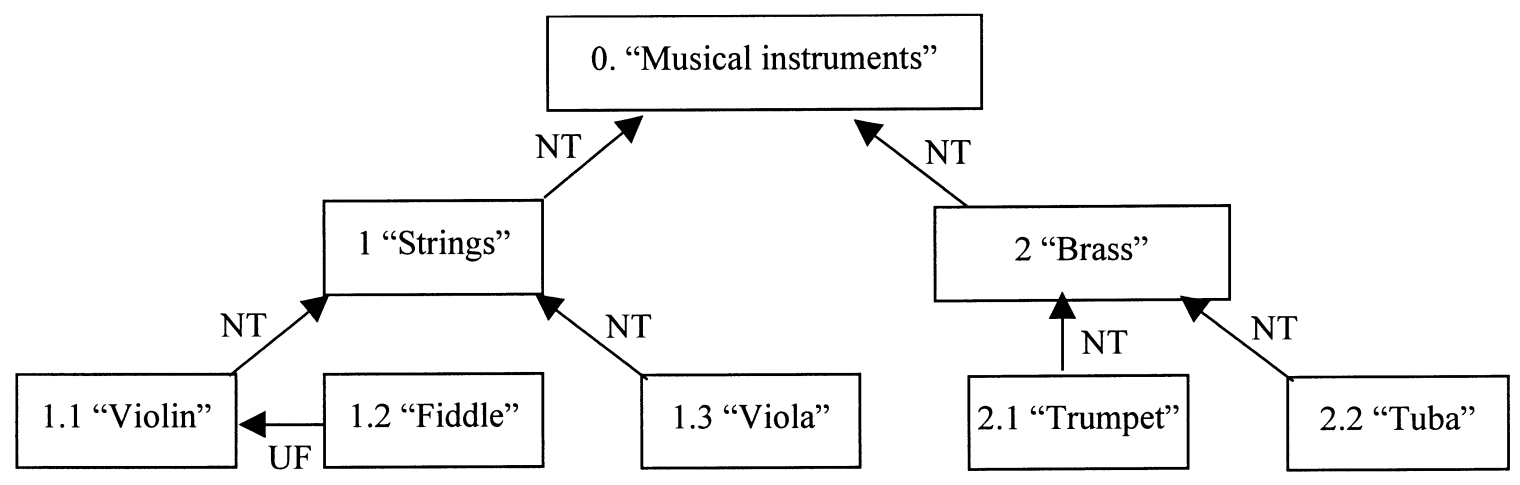

Figure 4. A controlled-term taxonomy of part of the Musical Instruments hierarchy.

\subsection{Taxonomies}

A taxonomy consists of a number of sound categories organised into a hierarchical tree. For example: voice, instruments, environmental sounds, animals, etc. Each of these classes can be broken down further into more detailed descriptions such as: female laughter, rain, explosions, birds, dogs, etc. Figure 4 shows musical instrument controlled terms that are organised into a taxonomy with 'Strings' and 'Brass'. Each term has at least one relation link to another term. By default, a contained term is considered a narrower term (NT) than the containing term. However, in this example, 'Fiddle' is defined as being nearly synonymous with, but less preferable than, 'Violin'. To capture such structure, the following relations are available as part of the ControlledTerm description scheme:

- BT - Broader term. The related term is more general in meaning than the containing term.

- NT-Narrower term. The related term is more specific in meaning than the containing term.

- US - Use. The related term is (nearly) synonymous with the current term but the related term is preferred to the current term.

- UF-Use for. Use of the current term is preferred to the use of the (nearly) synonymous related term.

- RT-Related Term. Related term is not a synonym, quasi-synonym, broader or narrower term, but is associated with the containing term.

The purpose of the taxonomy is to provide semantic relationships between categories. As the taxonomy gets larger and more fully connected, the utility of the category relationships increases. Figure 5 shows the taxonomy in figure 4 combined into a larger classification scheme, including animal sounds, musical instruments, Foley sounds (sound effects for film and television), and impact sounds. By descending the hierarchical tree, we find that there are seventeen leaf nodes in the taxonomy. By inference, a sound segment that is classified in one of the leaf nodes inherits the category label of its parent node in the taxonomy. For example, a sound classified as a 'Dog Bark' also inherits the label 'Animals'. We shall adhere to this taxonomy for illustrative purposes only; MPEG-7 allows full flexibility in defining taxonomies using controlled terms and can be used to define much larger taxonomies than the given example.

\subsection{Probability model classifiers}

A number of probability model description schemes are defined in MPEG-7. The motivation for standardisation is driven by the cost of designing and training a robust classifier, which can be very computationally intensive for large-scale applications. Statistical models for classification of content may be shared via a standard interface to the internal probability models. With these standardised schemes in hand, it is possible to share pretrained probability models between applications even if the specific extraction methods vary, thus allowing widespread reuse of models. The following sections outline the use of probability model description schemes for sound recognition.

\subsubsection{Finite state models}

Sound phenomena are dynamic. The spectral features vary in time and it is this variation that gives a sound its characteristic fingerprint for recognition. MPEG-7 sound recognition models partition a sound class into a finite number of states based on the spectral features; individual sounds are described by their trajectories through this state space. Each state is modelled by a continuous probability distribution such as a Gaussian.

The dynamic behaviour of a sound class through the state space is modelled by a $k \times k$ transition matrix that describes the probability of transition to each of the $k$ states in a model, given a current state. For a transition matrix, $\mathbf{T}$, the $i$ th row and $j$ th column entry is the probability of transitioning to state $j$ at time $t$ given state $i$ at time $t-1$.

An initial state distribution, which is a $k \times 1$ vector of probabilities, is also required for a finite-state model. The $k$ th element in the vector is the probability of being in state $k$ in the first observation frame. 


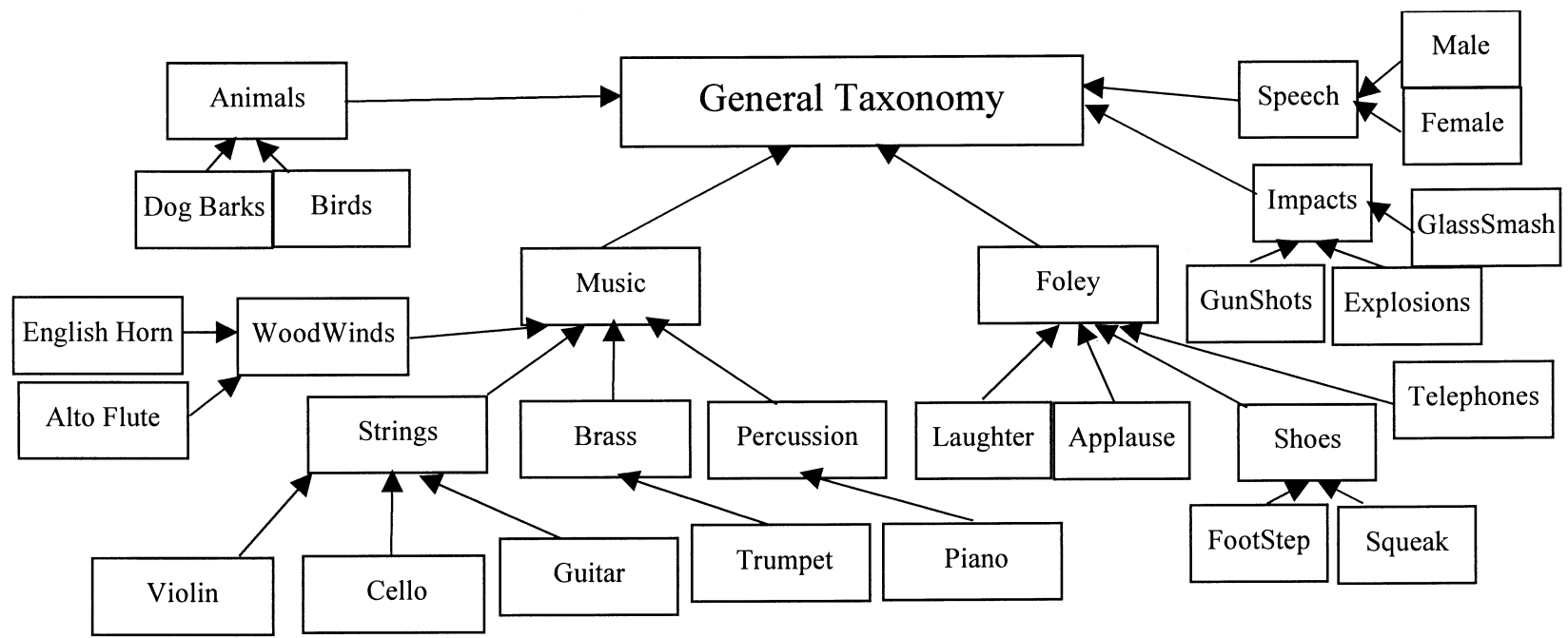

Figure 5. A hierarchical taxonomy including both musical and non-musical sources.

\subsubsection{Multi-dimensional Gaussian distributions}

The multi-dimensional Gaussian distribution is used for modelling the states. Gaussian distributions are parameterised by a $1 \times n$ vector of means, $\mathbf{m}$, and an $n \times n$ covariance matrix, $\mathbf{K}$, where $n$ is the number of features (columns) in the sound observation vectors. The expression for computation of probabilities for a random column vector, $\mathbf{x}$, given the Gaussian parameters, is:

$$
f_{\mathbf{x}}(\mathbf{x})=\frac{1}{(2 \pi)^{n / 2}|\mathbf{K}|^{1 / 2}} \exp \left[-\frac{1}{\mathbf{2}}(\mathbf{x}-\mathbf{m})^{T} \mathbf{K}^{-1}(\mathbf{x}-\mathbf{m})\right] .
$$

\subsubsection{Continuous hidden Markov models}

A continuous hidden Markov model is a finite state model with Gaussian distributions approximating each state's probability distribution. The states are hidden since we are not given the states along with the data. Rather, we must use the observable data to infer the hidden states. The states are clusters in the feature space of the sound data, namely, the SpectrumBasisProjection audio descriptor discussed earlier. Each row of the projected feature matrix, defined above, is a point in an $n$-dimensional vector space. The cloud of points is divided into multiple states (Gaussian clusters) using maximum a posteriori (MAP) estimation. The MAP estimator has the property of minimising the entropy, or degree of uncertainty, of the model whilst maximising the number of bits of evidence (information) that supports each model parameter (Brand 1998, 1999). Figure 6 shows a representation of four Gaussian-distributed states (vector point clouds) in two dimensions.

\subsubsection{MPEG-7 representation of hidden Markov models}

DDL example 3 illustrates the use of probability model description schemes for representing a continuous hidden Markov model with Gaussian states; in this example, floating-point numbers have been rounded to two decimal places for display purposes only.

\section{Sound Classification, Similarity and Example Search Applications}

\subsection{Classification application}

We trained nineteen HMMs, using MAP estimation, on a large database (2000+ sounds) divided into nineteen sound classes as described by the leaf nodes in the general sound taxonomy shown in figure 5 above. The database was split into separate training and testing data sets; i.e. $70 \%$ of the sounds were used for training the HMM models and $30 \%$ were used to test the recognition performance of the models on novel data. Each sound in the test set was presented to all nineteen models in parallel; the HMM with the maximum likelihood score, using a method called Viterbi decoding, was selected as the representative class for the test sound (see figure 7).

The results of classification performance on testing data are shown in table 1 . The results indicate very good recogniser performance across a broad range of sound classes. Of particular note is the ability of the classifiers to discriminate between speech sounds and non-speech sounds, including distinguishing between male and female speakers. The between- class discrimination indicates a high degree of category resolution for the system.

In addition to testing on the general sound taxonomy, we also conducted an experiment in music genre classification using the feature extraction and training methodology outlined above. We collected several hours of material from compact discs and MPEG-1 (Layer III) audio compressed files representing eight different musical genres. The data was split into $70 \% / 30 \%$ training/testing sets and the hidden Markov model classification system was trained as detailed above. Each sound 


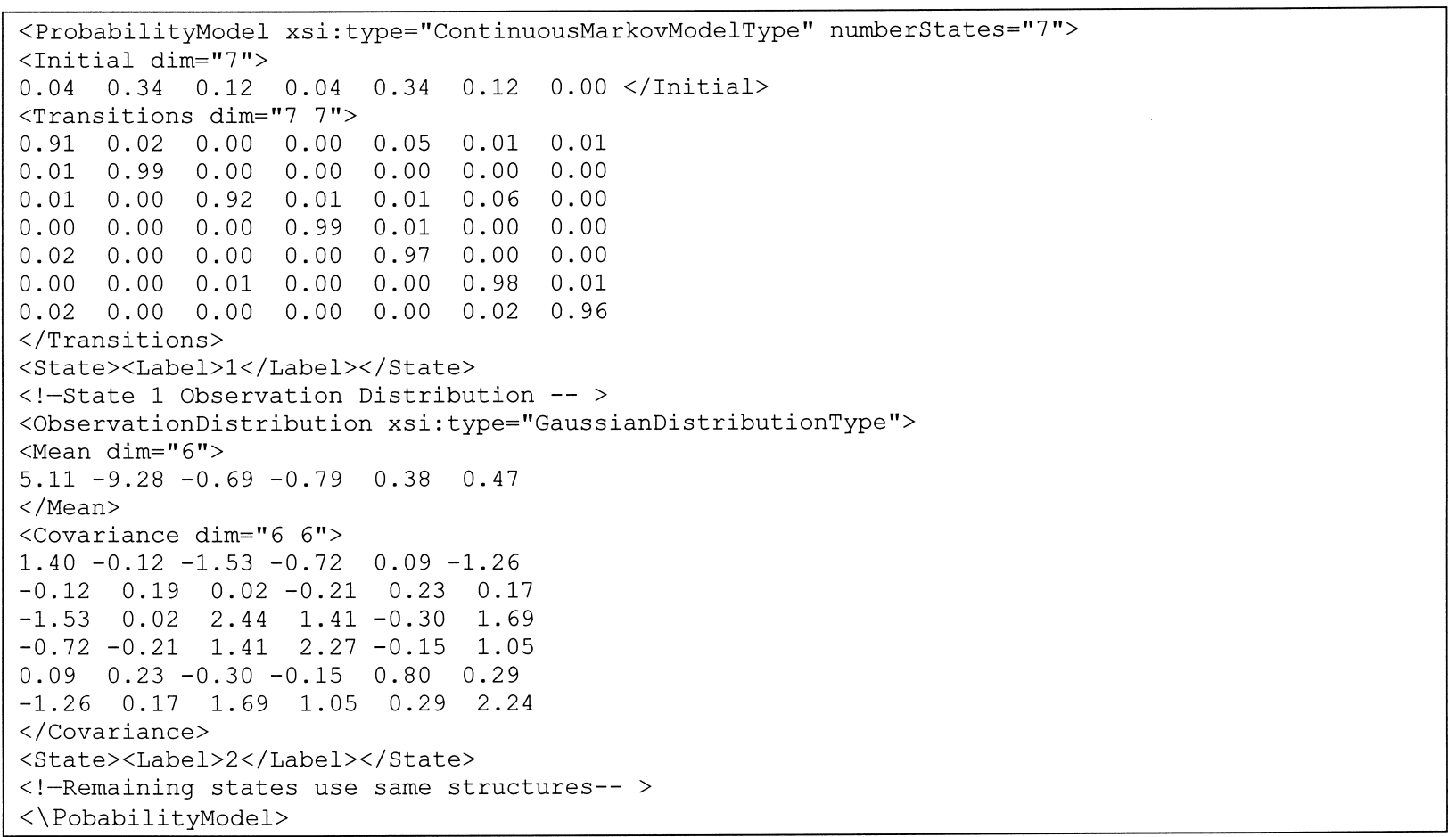

DDL Example 3. Instantiation of a Probability Model in the MPEG-7 DDL lanmguage. The model parameters were extracted using a maximum a posteriori estimator. The description scheme represents the initial state distribution, transition matrix, state labels, and individual Gaussian means and covariance matrices for the states.

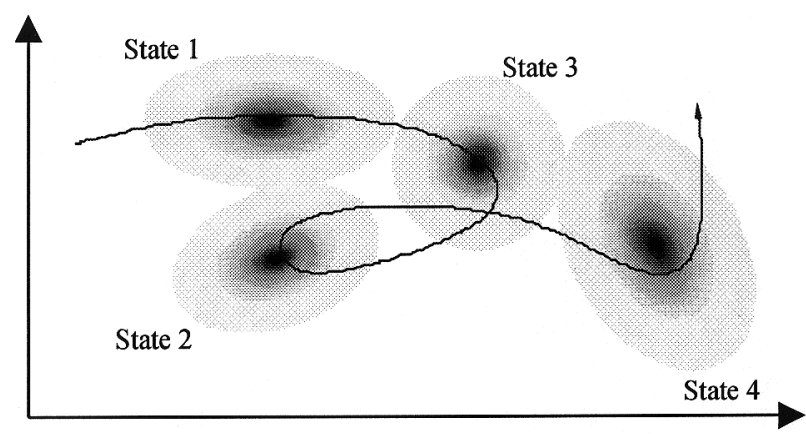

Figure 6. Four estimated Gaussian states depicted in a twodimensional vector space. Darker regions have higher probabilities. Sounds are represented as trajectories in such a vector space, the states are chosen to maximise the probability of the model given the observable evidence, i.e. the training data. The line shows a possible trajectory of a sound vector through the space.

file was split into separate 30 -second segments for training, thus the models were tuned to capture both local beat structure as well as phrase structures inherent within the data. Table 2 shows the results of classification of musical genres on novel musical sound file data. These results indicate the generalisation of the classification system to sounds that are composed of mixed sources with a high degree of internal structure.

\subsection{Generalised sound similarity}

In addition to classification, it is often useful to obtain a measure of how close two given sounds are in some perceptual sense. It is possible to leverage the internal, hidden, variables generated by an HMM in order to compare the evolution of two sounds through the model's state space. For each input query sound to a HMM, the output is a series of states through which sound passed. Each sampled state is given a likelihood that is used to cumulatively compute the probability that the sound actually belongs to the given model. The SoundModelstatePath descriptor contains the dynamic state path of a sound through a HMM model. Sounds are indexed by segmentation into model states or by sampling of the state path at regular intervals. Figure 8 shows a spectrogram of a dog bark sound with the state path through the 'DogBark' HMM shown below.

The state path is an important method of description since it describes the evolution of a sound with respect to physical states. The state path shown in the figure indicates physical states for the dog bark; there are clearly delimited onset, sustain and termination/silent states. This is true of most sound classes; the individual states within the class can be inspected via the state path representation and a useful semantic interpretation can often be inferred.

There are many possible methods for computing similarity between state paths; dynamic time warping and 


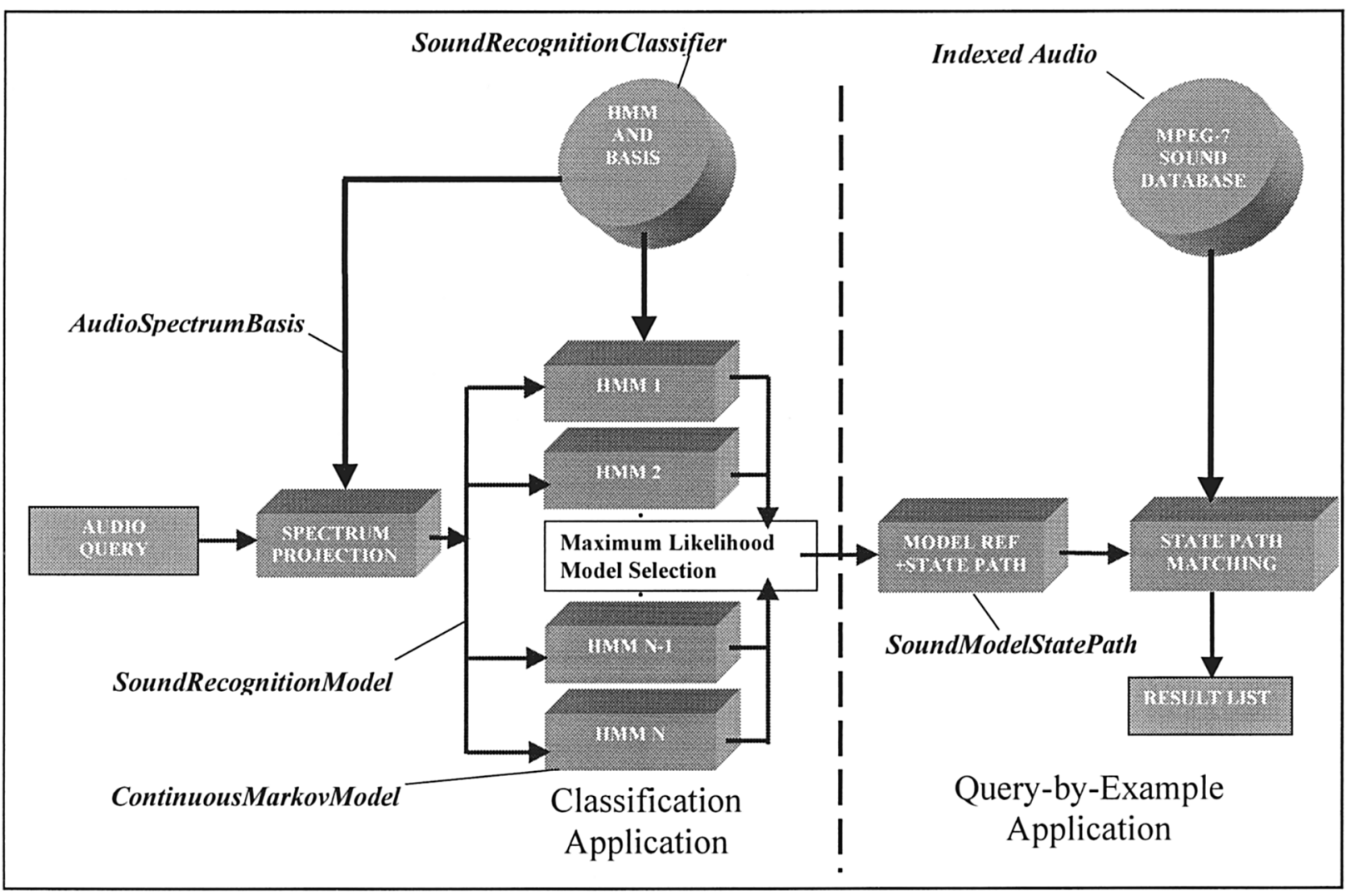

Figure 7. Sound classification and similarity system using parallel HMMs.
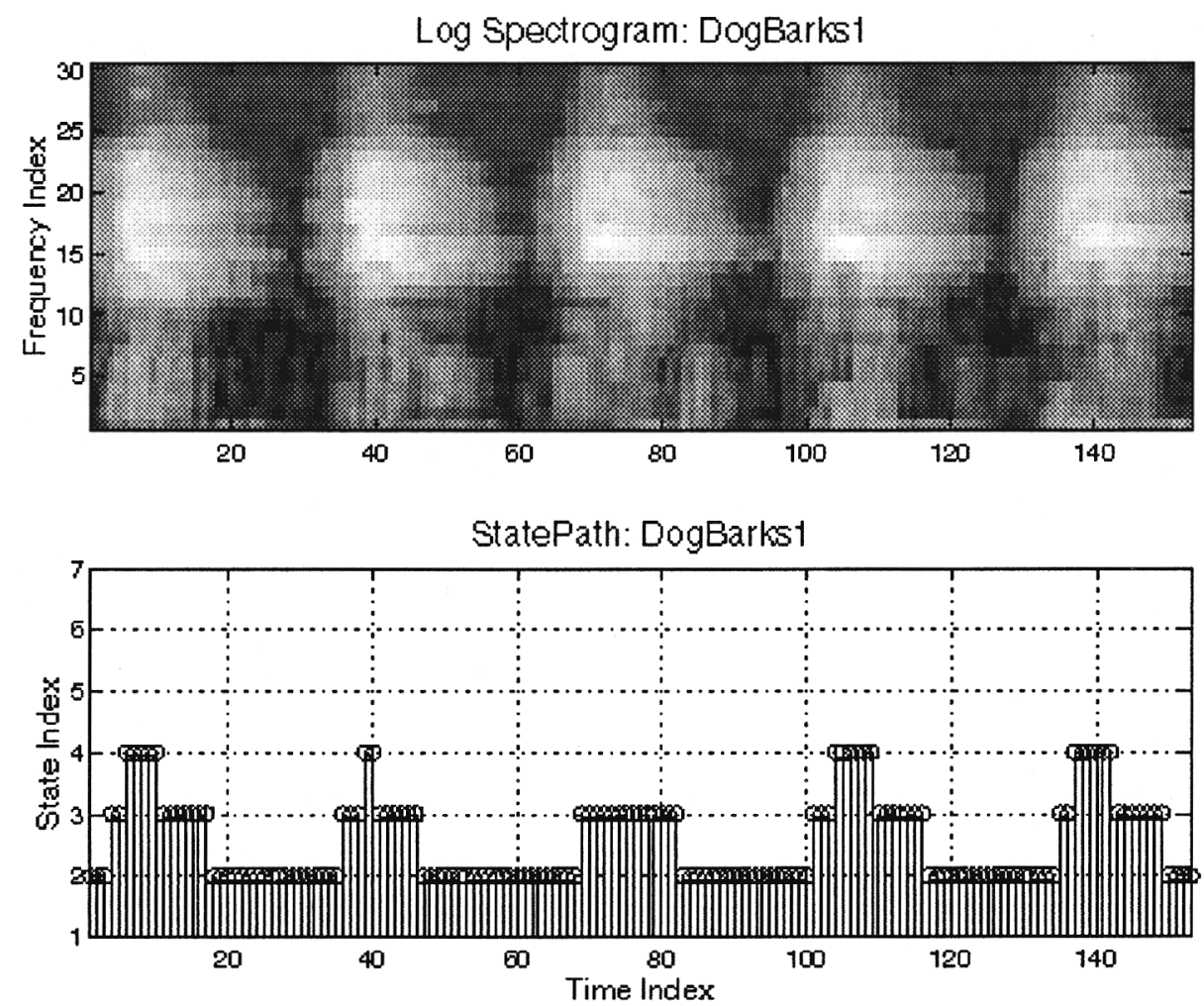

Figure 8. Dog bark spectrogram and the state path through the dog bark continuous hidden Markov model. 
Table 1. Performance of nineteen classifiers trained on $70 \%$ and cross-validated on $30 \%$ of a large sound database. The mean recognition rate indicates high recogniser performance across all the models.

\begin{tabular}{lc}
\hline Model name & \% correct classification \\
\hline [1] Alto Flute & 100.00 \\
[2] Birds & 80.00 \\
[3] Pianos (Bosendorfer) & 100.00 \\
[4] Cellos (Pizz and Bowed) & 100.00 \\
[5] Applause & 83.30 \\
[6] Dog Barks & 100.00 \\
[7] English Horn & 100.00 \\
[8] Explosions & 100.00 \\
[9] Footsteps & 90.90 \\
[10] Glass Smashes & 92.30 \\
[11] Guitars & 100.00 \\
[12] Gun shots & 92.30 \\
[13] Shoes (squeaks) & 100.00 \\
[14] Laughter & 94.40 \\
[15] Telephones & 66.70 \\
[16] Trumpets & 80.00 \\
[17] Violins & 83.30 \\
[18] Male Speech & 100.00 \\
[19] Female Speech & 97.00 \\
Mean recognition rate & 92.646 \\
\hline
\end{tabular}

Table 2. Performance of eight classifiers using a 70\%/30\% training/testing split for music genre classification.

\begin{tabular}{lc}
\hline Model name & $\begin{array}{c}\text { \% correct classification } \\
\text { (novel data) }\end{array}$ \\
\hline [1] Blugrass & 96.8 \\
[2] Reggae & 92.5 \\
[3] Rap & 100.00 \\
[4] Folk & 92.3 \\
[5] Blues & 98.7 \\
[6] Country & 88.9 \\
[7] Gospel & 95.7 \\
[8] New Age & 98.3 \\
Mean recognition rate & 95.4 \\
\hline
\end{tabular}

state histogram sum-of-square errors are two such methods. Dynamic time warping (DTW) uses linear programming to give a distance between two functions in terms of the cost of warping one onto the other. We may apply DTW to the state paths of two sounds in order to estimate the similarity of their temporal evolutions. However, there are many cases where the temporal evolution is not as important as the relative balance of occupied states between sounds. This is true, for example, with sound textures such as rain, clapping or crowd babble. For these cases it is preferable to use a temporally agnostic similarity metric such as the sum-ofsquare errors between state occupancy histograms. These similarity methods may be applied to a wide variety of sound classes and thus constitute a generalised sound similarity framework.

\subsection{Query-by-example application}

The system shown on the right-hand-side of figure 7 implements a query-by-example application. The audio feature extraction process is applied to a target query sound, namely spectrogram projection against a stored set of basis functions for each model in the classifier. The resulting dimension-reduced features are passed to a Viterbi decoder for the given classifier and the HMM with the maximum-likelihood score for the given features is selected. The model reference and state path are recorded and the results are matched by comparing the state path to the state paths of all the sounds for the given class in a pre-computed MPEG-7 index database.

Figure 9 shows a query sound (Laughter) and figure 10 shows the resulting closest matches using the difference in state-path occupancy histograms. The state paths and the histograms are also shown in the figures as well as the resulting distance estimates for each of the returned matches.

\subsection{Non-categorical similarity ratings}

Using such similarity measures it is possible to automatically organise sonic materials for a composition. The examples given above organise similarity rankings according to a taxonomy of categories. However, if a non-categorical interpretation of similarity is required, one may simply train a single HMM, with many states, using a wide variety of sounds. Similarity may then proceed without category constraints by comparing statepath histograms in the large generalised HMM state space.

\section{CONCLUSIONS}

In this paper we have outlined some of the tools that are available within the MPEG-7 standard for managing complex sound content. In the first part of the paper we presented independent subspace analysis as a method for performing analysis and re-synthesis of individual sources in a mixed audio file. We also showed that ISA may be used to obtain statistically salient features that may be applied with great generality to sound recognition and sound similarity tasks.

One of the major design criteria for the tools was the ability to analyse and represent a wide range of acoustic sources including textures and mixtures of sound. The tools presented herein exhibited good performance on musical sounds as well as traditionally non-musical sources such as vocal utterances, animal sounds, environmental sounds and sound effects. Amongst the applications presented were robust sound recognition using trained probability model classifiers and sound similarity matching using internal probability model state variables.

In conclusion, the description schemes and extractor 

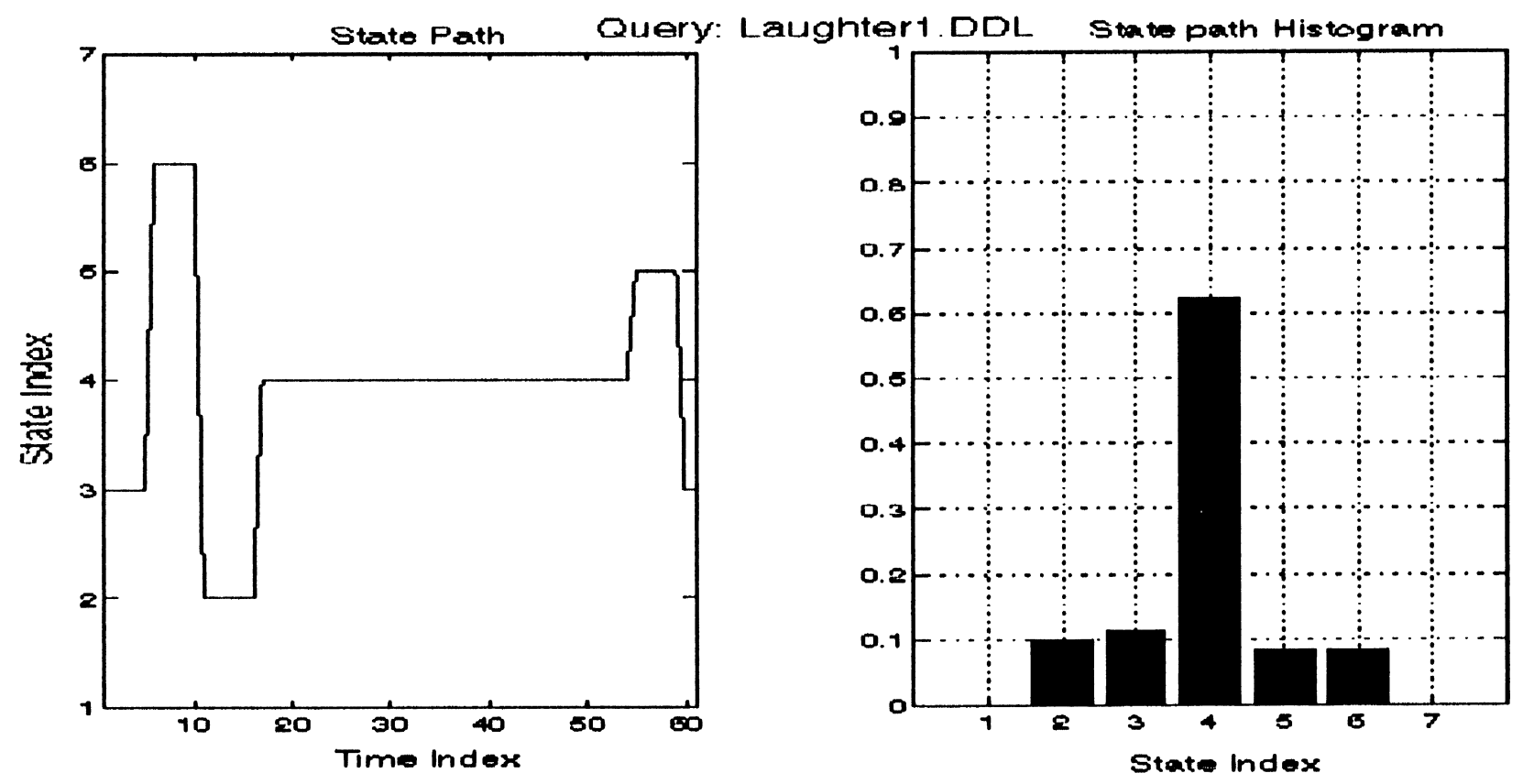

Figure 9. Query sound represented by a state-path histogram for the Laughter HMM.

Result List: Laughter1.DDL
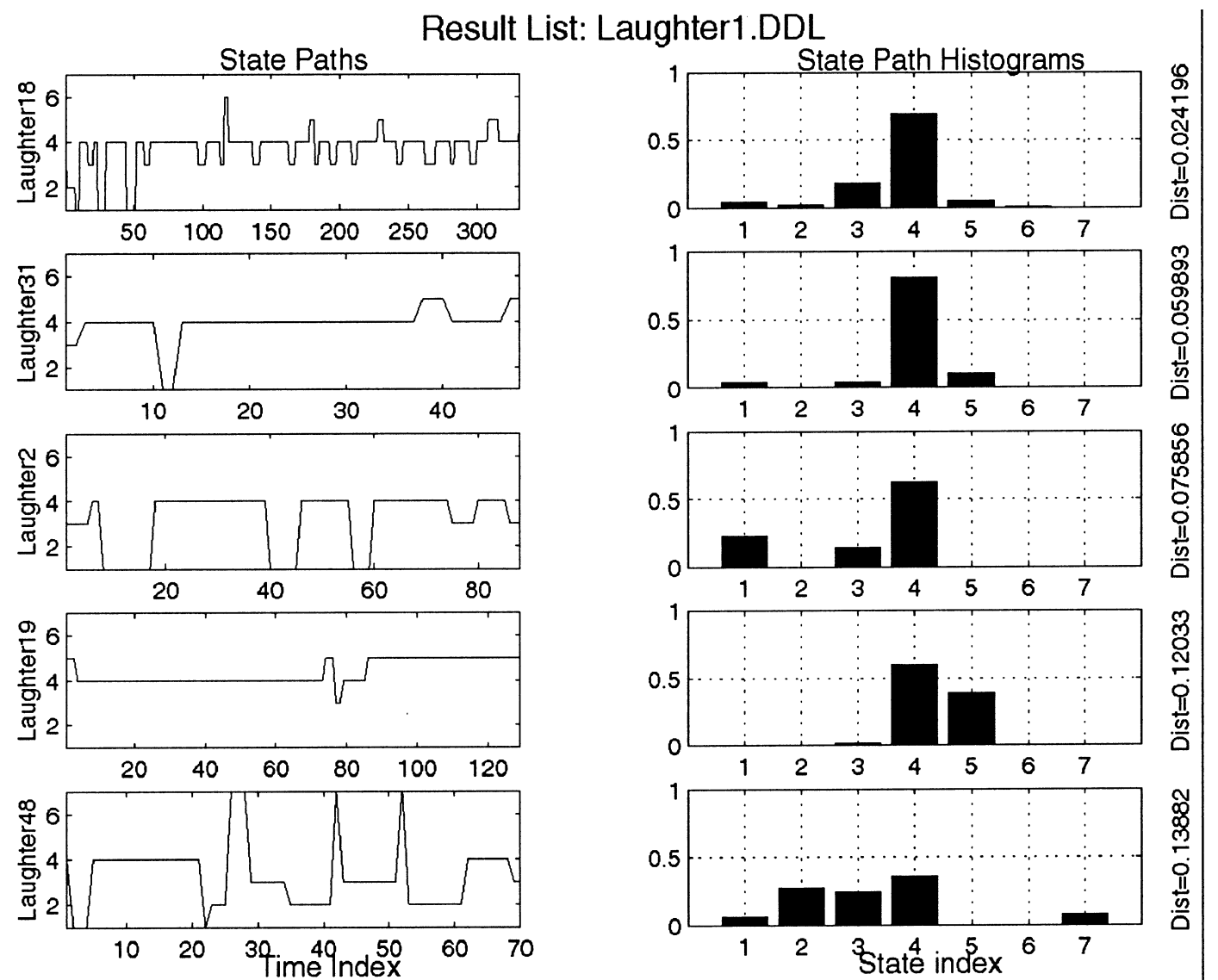

Figure 10. Five-best matches for the query sound. The distances between the target sound and the result sounds are given on the right-hand side of the figure. These distances were computed using the sum of square errors between the state-path histograms. 
methodologies outlined in this paper provide a consistent framework for analysing, indexing and querying sounds from a wide range of different classes. These tools have been made widely available as a component of the reference software implementation of the MPEG-7 standard. It is hoped that the ability to manipulate sound in novel ways and the ability to search for 'sounds like' candidates in a large database of sounds will become important new tools for sound-designers, composers and many other users of new music technology.

\section{REFERENCES}

Bell, A. J., and Sejnowski, T. J. 1995. An informationmaximization approach to blind separation and blind deconvolution. Neural Computation 7: 1,129-59.

Boreczky, J. S., and Wilcox, L. D. 1998. A hidden Markov model framework for video segmentation using audio and image features. In Proc. of ICASSP'98, pp. 3,741-4. Seattle, WA.

Brand, M. 1999a. Structure discovery in conditional probability models via an entropic prior and parameter extinction. Neural Computation 11(5): 1,155-83.

Brand, M. 1999b. Pattern discovery via entropy minimization. In Proc. Uncertainty' 99. Society of Artificial Intelligence and Statistics \#7, FL.

Cardoso, J. F., and Laheld, B. H. 1996. Equivariant adaptive source separation. IEEE Trans. On Signal Processing 4: $112-14$.
Casey, M. 2001a. MPEG-7 sound recognition tools. IEEE Trans. on Circuits and Systems Video Technology, Special Issue on MPEG-7. IEEE.

Casey, M. 2001b. Sound recognition tools. In B. S. Manjunath, P. Salembier and T. Sikora (eds.) Introduction to MPEG-7: Multimedia Content Description Language. J. Wiley.

Casey, M. 2001c. Reduced-rank spectra and entropic priors as consistent and reliable cues for general sound recognition. In Proc. of the Workshop on Consistent \& Reliable Acoustic Cues for Sound Analysis, September 2001.

Casey, M. A., and Westner, A. 2000. Separation of mixed audio sources by independent subspace analysis. In Proc. of the Int. Computer Music Conf. Berlin: ICMA.

ISO. 2002. Multimedia Content Description Interface. ISOIEC 15938-4 (Audio).

Hyvarinen, A. 1999. Fast and robust fixed-point algorithms for independent component analysis. IEEE Trans. On Neural Networks 10(3): 626-34.

Martin, K. D., and Kim, Y. E. 1998. Musical instrument identification: a pattern-recognition approach. Presented at the 136th Meeting of the Acoustical Society of America, Norfolk, VA.

Wold, E., Blum, T., Keislar, D., and Wheaton, J. 1996. Content-based classification, search and retrieval of audio. IEEE Multimedia (Fall issue): 27-36.

Zhang, T., and Kuo, C. 1998. Content-based classification and retrieval of audio. SPIE 43rd Annual Meeting, Conf. on Advanced Signal Processing Algorithms, Architectures and Implementations VIII. San Diego, CA. 


\section{Music from Cambridge}

\section{Popular Music}

Popular Music is an international multi-disciplinary journal covering all aspects of the subject from the formation of social group identities through popular music, to the workings of the global music industry, or how particular pieces of music are put together. Relating to any kind of popular music, from the global commercial sphere to local folk or traditional music from any historical era or geographical location, the journal carries articles by scholars from a large variety of perspectives. These include the anthropology of music, cultural studies, ethnomusicology, feminism, gender studies, geography, history, literature studies, media studies, musicology, sociology and any other disciplines or cross-disciplines that can be shown to have relevance to the subject. Each issue contains substantial, authoritative and influential articles, shorter topical pieces, and reviews of a wide range of books. Presenting scholarly debate and analysis, the journal keeps readers up to date with the latest developments in popular music research.

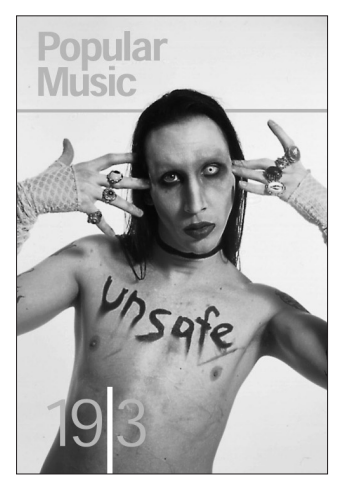

Some issues are thematic, of which recent examples include the musical construction of place; the characteristics, influences and social significance of New Orleans music; and special issues on gender, sexuality and popular music, and on music and the media are forthcoming. An annual annotated book list of popular music titles from around the world is also included.

\section{Online Access}

The journal is online at Cambridge Journals Online.

Visit the journal homepage for free access to abstracts and tables of contents.

\section{http://journals.cambridge.org/journal_popularmusic}

\section{Subscriptions}

Volume 21 in 2002: January, May and October

Institutions print and electronic: $\mathrm{f} 87 / \$ 136$

Institutions electronic only: $\mathrm{f} 82 / \$ 130$

Individuals print only: $£ 40 / \$ 60$

Students: $£ 31 / \$ 50$

International Association for the Study of Popular Music: $£ 31 / \$ 50$

Print ISSN 0261-1430

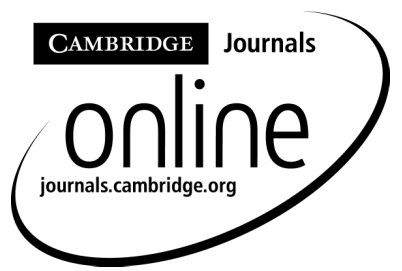

to contact the Customer Services Journals Division -

in Cambridge: tel +44 (0)1223 326070 fax +44 (0)1223 325150 email journals@cambridge.org in New York: tel (914) 9379600 fax (914) 9374712 email subscriptions_newyork@cambridge.org 


\section{Music from Cambridge}

\section{Plainsong and Medieval Music}

Published twice a year in association with the Plainsong and Mediaeval Music Society, this journal covers the entire field of plainchant and medieval music. It encompasses Eastern and Western chant, secular lyric, music theory and paleography, performance practice, as well as medieval polyphony, sacred and secular. The chronological scope extends from late antiquity to the early Renaissance and to the present day in the case of chant. In addition to articles embodying original research, the journal publishes book reviews, a list of important recent publications, an annual bibliography of chant research and an annual discography of chant recordings.

\section{Online Access}

The journal is online at Cambridge Journals Online.

Visit Cambridge Journals for free access to abstracts and tables of contents.

\section{http://journals.cambridge.org}

\section{Subscriptions}

Volume 11 in 2002: April and October

Institutions print and electronic: $\mathrm{f} 56 / \$ 88$

Institutions electronic only: $£ 52 / \$ 82$

Individuals print only: $£ 30 / \$ 45$

Association Paid Member rate $\mathrm{f19/ \$}$ Plainsong and Mediaeval

Music Society

Print ISSN 0961-1371

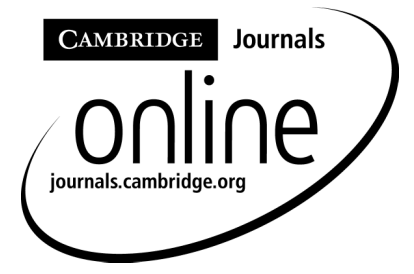

to contact the Customer Services Journals Division -

in Cambridge: tel +44 (0)1223 326070 fax +44 (0)1223 325150 email journals@cambridge.org

in New York: tel (914) 9379600 fax (914) 9374712 email subscriptions_newyork@cambridge.org 


\section{Music from Cambridge}

\section{British Journal of Music Education}

$B J M E$ aims to provide clear, stimulating and readable accounts of current issues in music education worldwide, together with a section containing extended and useful book reviews. In particular, it strives to strengthen professional development and improve practice within the field of music education. The range of subjects covers classroom music teaching, individual instrumental teaching and vocal teaching, music in higher education, international comparative music education, the development of literature in this field, and teacher education. The journal is fully refereed and contributors include practising teachers from schools, colleges and universities. Audio examples are occasionally supplied on a CD with no extra charge.

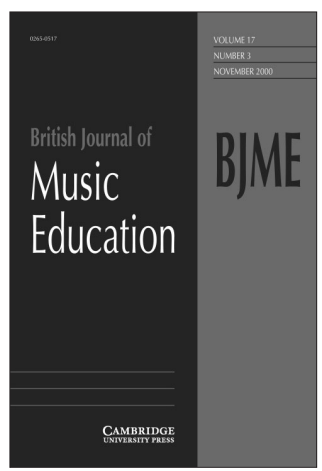

Recent articles have covered such topics as the teaching of music by non-specialists in primary schools, the uses of information technology in music lessons, and musical education in Africa.

\section{Online Access}

The journal is online at Cambridge Journals Online.

Visit Cambridge Journals for free access to abstracts and tables of contents.

\section{http://journals.cambridge.org}

\section{Subscriptions}

Volume 19 in 2002: March, July and November

Institutions print and electronic: $\mathrm{f} 69 / \$ 108$

Institutions electronic only: $f 64 / \$ 100$

Individuals print only: $€ 36 / \$ 57$

Students: $f 25 / \$ 42$

Probationary teachers: $\mathrm{f25/} \$ 42$

Print ISSN 0265-0517

Electronic ISSN 1469-2104

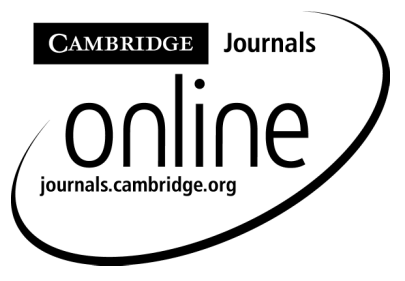

to contact the Customer Services Journals Division -

in Cambridge: tel +44 (0)1223 326070 fax +44 (0)1223 325150 email journals@cambridge.org

in New York: tel (914) 9379600 fax (914) 9374712 email subscriptions_newyork@cambridge.org 